\title{
Effect of Indigestible Polysaccharides on Pancreatic Exocrine Secretion and Biliary Output
}

\author{
Sachie IKEgAMI, ${ }^{1}$ Hirokazu HARAdA, ${ }^{2}$ Noboru TsuchIHASHI, ${ }^{3}$ \\ Sumiko NAGAYAMA, ${ }^{4}$ Eiichi Nishide, ${ }^{2}$ and Satoshi INNAMI ${ }^{1 *}$ \\ ${ }^{1}$ Division of Food Science, National Institute of Nutrition, \\ Shinjuku-ku, Tokyo 162, Japan \\ ${ }^{2}$ Department of Fisheries, College of Agriculture and \\ Veterinary Medicine, Nihon University, \\ Setagaya-ku, Tokyo 154, Japan \\ ${ }^{3}$ Department of Nutrition. Chiba College of Health Science, \\ Chiba, Chiba 260, Japan \\ ${ }^{4}$ Tokyo Kaseigakuin College, Chiyoda-ku, Tokyo 100, Japan
}

(Received August 29, 1983)

\begin{abstract}
Summary The present study was designed to determine the effect of purified viscous polysaccharides on pancreatic exocrine secretion and biliary output. Male Sprague-Dawley rats aged 5 weeks were fed either on the control diet which contained no fiber or on the experimental diets that contained $5 \%$ indigestible polysaccharides (agar, $\kappa$-carrageenan, $\mathrm{Na}$ alginate and konjac mannan). After pretreatment with each diet for 2 weeks, the diet was discontinued overnight, and the animals were then allowed to eat $4 \mathrm{~g}$ of the test diet in $1 \mathrm{~h}$. Immediately after this, a polyethylene tube was placed in the common bile duct, under anesthesia. Combined, free drained pancreatic-bile juice was collected for the following $1 \mathrm{~h}$.

Long-term (2 weeks) administration of Na-alginate and konjac mannan gave rise to increases in the volume of secreted juice and in the output of total bile acids, amylase and protease, whereas that of agar and $\kappa$ carrageenan had no affect. Since a single dose of Na-alginate and konjac mannan was ineffective as regards pancreatic-bile secretion, the elevated secretion observed over prolonged periods may result from processes of adaptation against highly viscous polysaccharides.
\end{abstract}

Key Words indigestible polysaccharides, agar, $\kappa$-carrageenan, Naalginate, konjac mannan, pancreatic secretion, biliary output, viscosity

Dietary fibers are known to increase the fecal excretion of protein, fat and other nutrients $(1,2)$, which is probably due to the interference with digestion and absorption of food in the gut (3). In a previous paper, we reported that the digestive

${ }^{1}$ 池上幸江, ${ }^{2}$ 原田広和, ${ }^{3}$ 土橋 昇, $^{4}$ 永山すみ子, 西出英一, ${ }^{1}$ 印南 敏 
organs responded with increased weight and length to oral administration of dietary fibers in experimental animals (4), and that some highly viscous indigestible polysaccharides such as $\mathrm{Na}$-alginate, $\kappa$-carrageenan, pectin and konjac mannan were able to affect the digestive function of rats. Sheard and Schneeman (5) observed that some dietary fibers led to an increase in pancreas weight and to stimulation of digestive enzyme secretion from the pancreas, although the latter response was shown with wheat bran and was indirectly determined.

This study was undertaken to determine the effect of viscous indigestible polysaccharides on pancreatic exocrine secretion and biliary output by an experimentally designed direct method.

\section{METHODS}

1. Animals. Weanling male Sprague-Dawley rats aged 5 weeks were used in all experiments. Rats were housed individually in stainless-steel wire-bottomed cages and kept at a constant temperature of $23 \pm 1^{\circ} \mathrm{C}$ and a $12 \mathrm{~h}$ light-dark cycle.

2. Diet. A basal diet which consisted of casein $20 \%$, soybean oil $10 \%$, mineral mixture (AIN-76(6)) 4.0\%, vitamin mixture (AIN-76(6)) 1.0\%, choline bitartrate $0.15 \%$, and sucrose up to $100 \%$ was used as the control non-fiber diet.

Rats were fed on either the control diet or one of the four experimental diets in which an equal amount of sucrose at the $5 \%$ level was substituted with the following samples: agar (agar powder, Wako Pure Chemical Ind., Ltd.), $\kappa$-carrageenan (Wako Pure Chemical Ind., Ltd.), Na-alginate (Wako Pure Chemical Ind., Ltd.) and konjac mannan (konjac flour rinsed with $50 \%$ ethyl alcohol several times).

3. Collection of pancreatic-biliary juice. Rats were maintained on each of the diets and water ad libitum for given periods, being fasted one night before the experiment. On the morning of the experiment, animals were allowed to eat $4 \mathrm{~g}$ of the same diet in $1 \mathrm{~h}$. Anesthesia was given by an intraperitoneal administration of urethane at $130 \mathrm{mg} / 100 \mathrm{~g}$ body weight. The animals were then operated on and a pancreatic-bile cannulae of polyethylene tubing was inserted through the common bile duct near the duodenum. The secreted juice was free drained and collected for $1 \mathrm{~h}$.

4. Analytical method. The volume of juice obtained was measured. Protein concentration and amylase activity in the juice was determined according to the methods of Lowry (7) and Fuwa (8) respectively. Protease activity was assayed by Anson's method(9) using urea-denaturated hemoglobin as the substrate after activating with enterokinase prepared from the rat duodenal mucosa. Total bile acid content was assayed using $3 \alpha$-hydroxysteroid dehydrogenase (10).

5. Statistics. Data obtained from 6 or 7 rats on each experimental diet were compared with the data from those on the control diet by using Student's nonpaired $t$-test. 


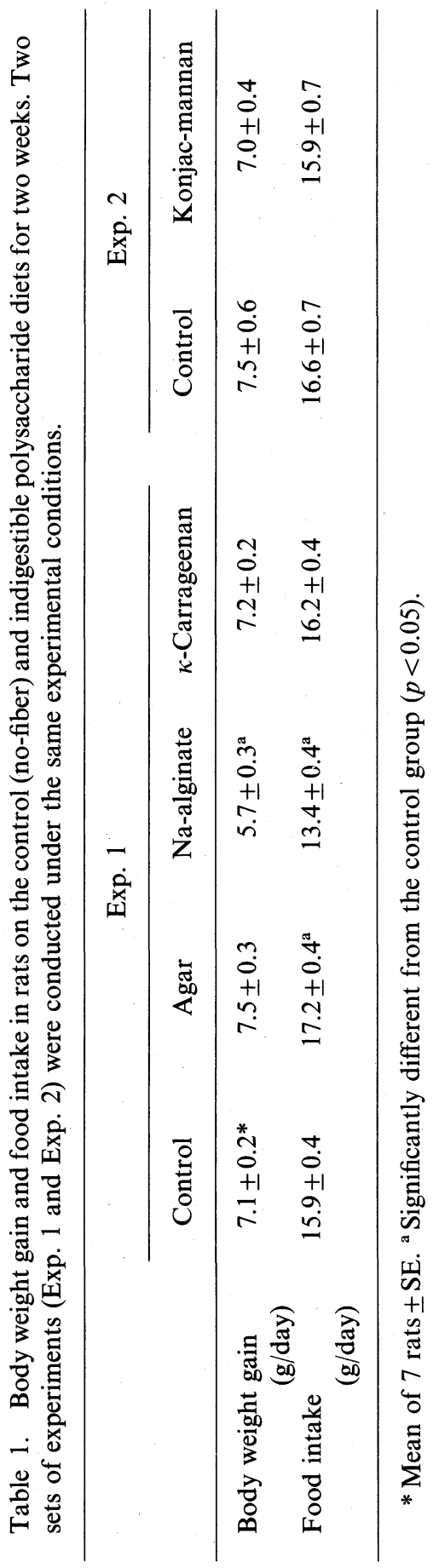

Vol. 30, No. 6, 1984 


\section{RESULTS}

1. Response of pancreatic-biliary secretion after two weeks of continuous administration of polysaccharides

Experiments 1 and 2 were performed separately under the same conditions to clarify the effect of seaweed polysaccharides and konjac mannan. Table 1 shows the results of averaged daily body weight gain and food consumption in rats fed on the control and indigestible polysaccharide diets for 2 weeks. All rats on the agar, $\kappa$ carrageenan or konjac mannan diet showed nearly the same body weight gain and food consumption as each control group of rats. However, the rats on $\mathrm{Na}$-alginate diet showed significantly low values as compared to the control.

The pancreatic-biliary responses determined 2 weeks after the feeding of experimental diets are shown in Fig. 1. The animals fed $\mathrm{Na}$-alginate showed increases in the volume of secreted juice and in the outputs of total bile acids,

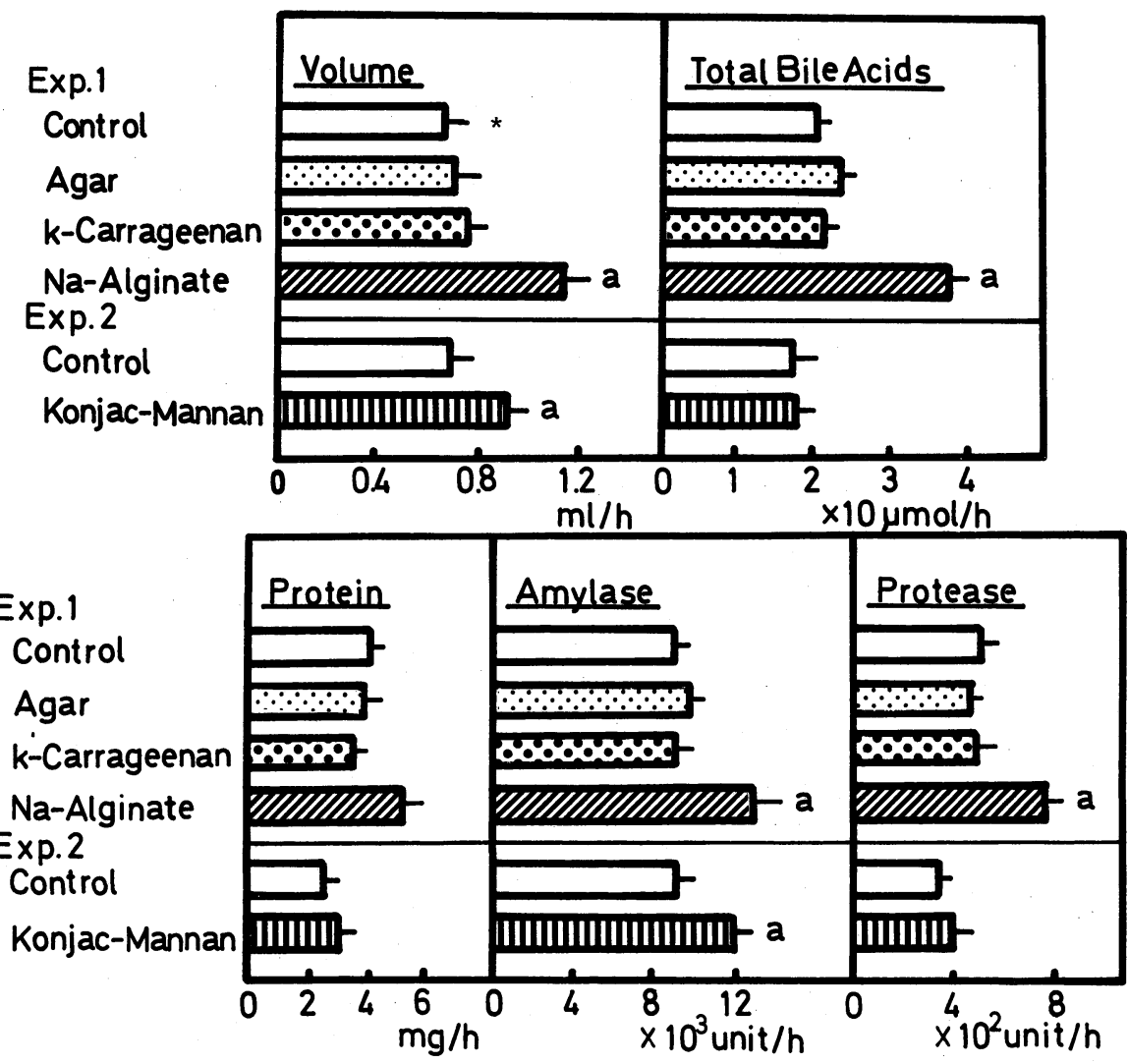

Fig. 1. Volume and composition of pancreatic-bile juice after two weeks continuous administration of indigestible polysaccharides. See footnote in Table $1 .{ }^{*}$ Means of 7 rats \pm SE. a Significantly different from the control group $(p<0.05)$. 


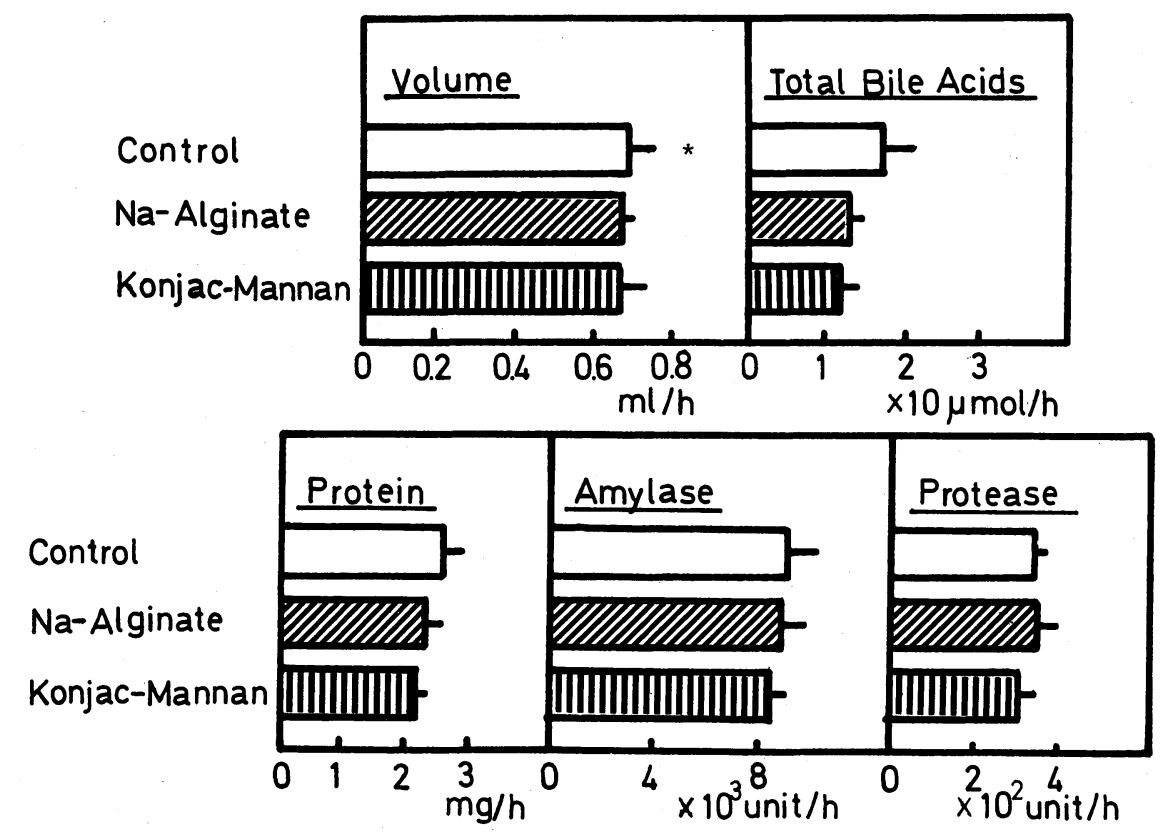

Fig. 2. Volume and composition of pancreatic-bile juice after single administration of indigestible polysaccharides. * Means of 6 rats \pm SE. ${ }^{\text {a }}$ Significantly different from the control group $(p<0.05)$.

amylase and protease as compared to the control. The animals on konjac mannan in experiment 2 also responded with an elevated juice volume and amylase secretion and without any changes in the secretion of total bile acids and protease.

On the other hand, the two groups of animals fed agar and $\kappa$-carrageenan did not show any significant changes from the control in the pancreatic-bile secretion.

2. Response of pancreatic-bile secretion to single administration of Na-alginate or konjac mannan

As an enhanced pancreatic-bile response was obtained in the above experiments in which either Na-alginate or konjac mannan was given, another experiment was performed to see whether such a response could be induced immediately after single administration of those polysaccharides.

Figure 2 shows pancreatic-bile secretion following single administration of the control, Na-alginate or konjac mannan diets. All the rats used in this experiment were given the control diet for 2 weeks before the study. No enhanced secretory response to either $\mathrm{Na}$-alginate or konjac mannan was observed when compared to the control.

\section{Serial determinations on the responses to Na-alginate feeding}

Table 2 shows the results obtained before and 3, 7 and 14 days after $\mathrm{Na}$ Vol. 30, No. 6, 1984 


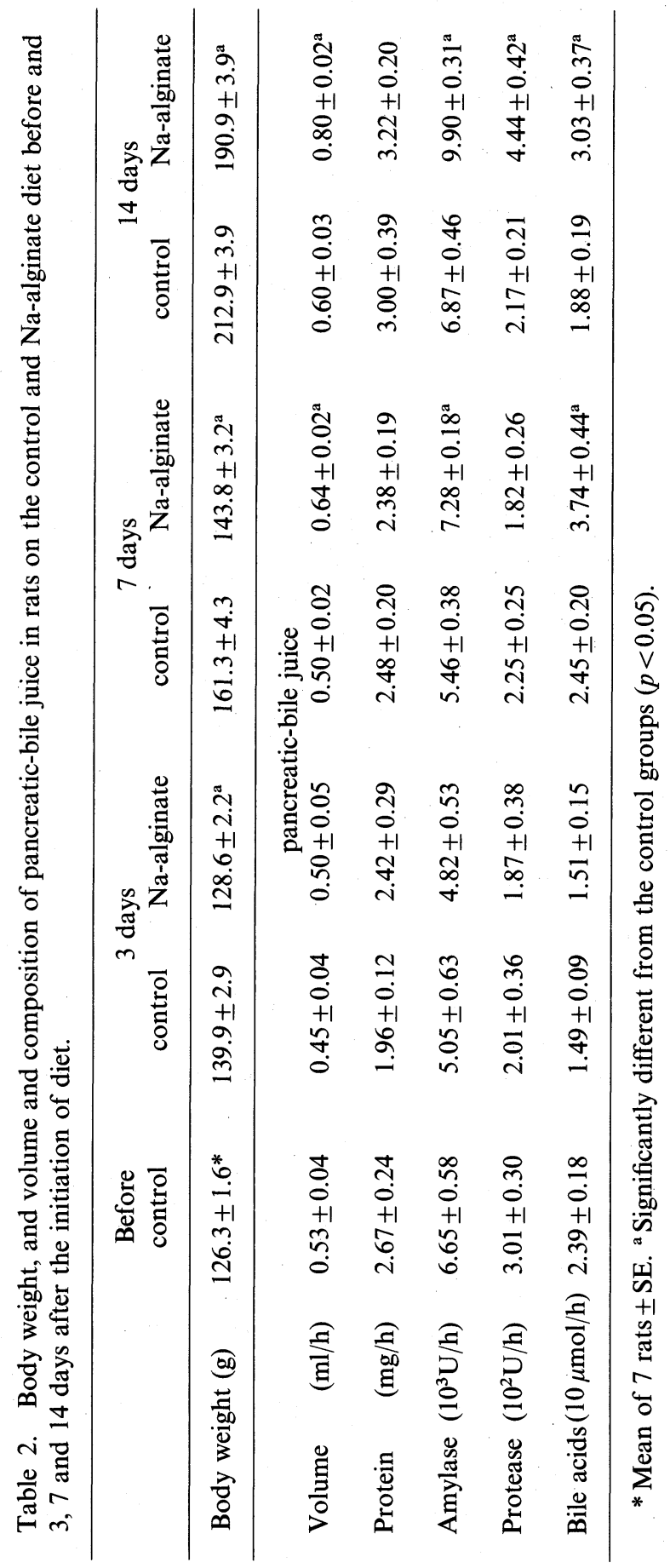


alginate feeding. As expected, the animals on Na-alginate had suppressed body weight compared to the control animals. The volume of juice, amylase activity and total bile acids in the pancreatic-bile secretion were significantly more increased 7 days after the $\mathrm{Na}$-alginate feeding than in the control group, whereas an increase in protease activity seemed to take more than 7 days. Administration of $\mathrm{Na}$-alginate for 14 days brought about a more marked rise in the volume of juice and amylase activity, but did not further increase the secretion of total bile acids.

\section{DISCUSSION}

As shown in our previous report (4), certain indigestible polysaccharides seem to have an interfering action with the digestion and absorption of nutrients in the gut. In this study, the result of $\mathrm{Na}$-alginate and konjac mannan feeding showed an increased secretion of pancreatic-biliary juice not only in the volume excreted but also for amylase activity and total bile acids. No significant effect was obtained from either agar or $\kappa$-carrageenan feeding.

Kanno(11) postulated that the stimulation of pancreatic exocrine secretion would occur in two phases: first, in the elimination phase and second, in the intakesynthesis phase. The former occurs in seconds or minutes, while the latter occurs after weeks, months or years. According to this concept, there seems to be present at least the two mechanisms by which pancreatic exocrine and biliary secretion can be increased. It is well known that the components of digested food such as essential amino acids or fatty acids as well as secreted gastric acid are the potent stimuli in releasing both cholecystokinin and secretin from the small intestine (12). However, this was not found to be the case in the present study, because, as shown in Fig. 2, single administration of each indigestible polysaccharide failed to increase the pancreatic-bile secretion. Schneeman (13) also reported that a continuous duodenal infusion of indigestible polysaccharides such as cellulose or pectin in conscious rats failed to stimulate pancreatic protein secretion. Therefore, the enhanced pancreaticbile output observed after prolonged administration of indigestible polysaccharides would not be mediated through the release of cholecystokinin or secretin.

The second mechanism involves responding or adapting to long-term administration of the test diets. It is known that the pancreas may develop adaptive changes either morphologically or functionally. An increase in pancreas weight following administration of dietary fibers has been described before (4). As to the functional changes, it is also known that an administration of carbohydrate, fat or protein-rich diet generally leads to an elevation of amylase, lipase or protease activity in pancreatic juice, respectively (14-17). In this study, 5\% carbohydrate was substituted with polysaccharides which was present in the diet at a low level. However, the results appeared sufficient to indicate an increase in pancreatic-bile secretion, when Na-alginate or konjac mannan was used for prolonged periods. It has been shown that a similar type of pancreatic juice secretion occurs when trypsin inhibitors are orally administrated to rats (18-21). In those experiments, trypsin 
inhibitors induced not only pancreatic hypertrophy but also increased pancreatic enzyme secretion in rats. Trypsin inhibitors are known to bind trypsin, lower the enzymatic activity of trypsin and interfere with protein digestion in the small intestine.

Green and Lyman (20), Green et al. (22) and Miyasaka and Green (23) reported that the secretion of the pancreatic enzymes increased shortly after remove of pancreatic-bile juice from the intestine. They proposed that trypsin and/or chymotrypsin depress pancreatic enzyme secretion by means of negative feedback control.

Green and Nasset (24) observed that the presence of bile protected pancreatic enzyme activities in the intestine and regulated pancreatic biliary output. They $(20,24)$ suggested that protease and bile acids might have a role in the release of humoral factors existing in the mucosa of the intestinal wall.

As mentioned earlier(4), both $\mathrm{Na}$-alginate and konjac mannan are more viscous than the other polysaccharides. The results of this experiment showed that these highly viscous polysaccharides might have slowed the rate of diffusion of the enzymes and nutrients in the gut, thereby lowering the rate of digestion and absorption.

Since Dooley and Valenzuela(25) reported that in human duodenum the receptors which are responsible for the volume and osmolarity changes in the intraluminal contents exist to regulate both pancreatic enzymes and bicarbonate secretion, the enhanced pancreatic function observed in this experiment seemed to be partially induced by the prolonged mechanical stimulation of the undigested foods.

As Na-alginate is more hydrophilic and viscous than konjac mannan, it is suggested that the gastric emptying of the polysaccharide-containing foods takes more time in the Na-alginate group of animals than in the konjac mannan group. Therefore, this delayed gastric emptying was thought to be responsible for the reduced food intake and the reduction of body weight gain in the Na-alginate group.

\section{REFERENCES}

1) Beyer, P. L., and Flynn, M. A. (1978): Effects of high- and low-fiber diets on human feces. J. Am. Diet. Assoc., 72, 271-277.

2) Southgate, D. A., and Durnin, J. V. G. A. (1970): Calorie conversion factors. An experimental reassesment of the energy value of human diets. Br. J. Nutr., 24, 517-535.

3) Kelsay, J. L., Behall, K. M., and Prather, E. S. (1978): Effect of fiber from fruits and vegetables on metabolic responses of human subjects. 1. Bowel transit time, number of defecations, fecal weight, urinary excretion of energy and nitrogen and apparent digestibilities of energy, nitrogen and fat. Am. J. Clin. Nutr., 31, 1149-1153.

4) Ikegami, S., Tsuchihashi, N., Nagayama, S., Harada, H., Nishide, E., and Innami, S. (1983): Effect of indigestible polysaccharides on function of digestion and absorption in rats. Nippon Eiyō Shokuryō Gakkaishi (J. Jpn. Soc. Nutr. Food Sci.), 36, 163-168.

5) Sheard, N. F., and Schneeman, B. O. (1980): Wheat bran's effect of digestive enzyme activity and bile acid levels in rats. J. Food Sci., 45, 1645-1648. 
6) Report of the American Institute of Nutrition; Ad Hoc Committee on Standards for Nutritional Studies (1977): J. Nutr., 107, 1340-1348.

7) Lowry, O. H., Rosebrough, N. J., Farr, A. L., and Randall, R. J. (1951): Protein measurement with the Folin phenol reagent. J. Biol. Chem., 193, 265-275.

8) Fuwa, H. (1954): A new method for microdetermination of amylase activity by the use amylose as the substrate. J. Biochem., 41, 583-603.

9) Anson, M. L. (1938): The estimation of pepsin, trypsin, papain, and cathepsin with hemoglobin. J. Gen. Physiol., 22, 79-89.

10) Turley, S. D., and Dietschy, J. M. (1978): Re-evaluation of the $3 \alpha$-hydroxysteroid dehydrogenase assay for total bile acids in bile. J. Lipid Res., 19, 924-928.

11) Kanno, T. (1975): Secretory mechanisms of the exocrine pancreas. Metab. Dis., 12, $1115-1120$.

12) Wang, C. C., and Grossman, M. I. (1951): Physiological determination of release of secretin and pancreozymin from intestine of dogs with translated pancreas. Am. J. Physiol., 164, 527-545.

13) Schneeman, B. O. (1979): Acute pancreatic and biliary response to protein, cellulose and pectin. Nutr. Rep. Int., 20, 45-48.

14) Abdeljlil, A. B., and Desnuelle, P. (1964): The adaptation of the exocrine enzymes of rat pancreas to the composition of the diet. Biochim. Biophys. Acta, 81, 136-149.

15) Johnson, A., Hurwitz, R., and Kretchmen, N. (1977): Adaptation of rat pancreatic amylase and chymotrypsinogen to changes in diet. J. Nutr., 107, 87-96.

16) Howard, F., and Yudkin, J. (1963): Effect of dietary change upon the amylase and trypsin activities of the rat pancreas. Br. J. Nutr., 17, 281-294.

17) Hulan, H. W., and Bird, F. H. (1972): Effect of fat level in isonitrogenous diets on the composition of avan pancreas juice. J. Nutr., 102, 459-468.

18) Snook, J. T. (1969): Factors in whole-egg protein influencing dietary induction of increases in enzymes and RNA levels in rat pancreas. J. Nutr., 97, 286-294.

19) Macleod, E. P., Derbenwick, J. P., and Snook, J. T. (1972): Comparison of pancreatic changes induced in rat soybean and egg-white trypsin inhibitors fed with different levels of nitrogen. J. Nutr., 102, 469-478.

20) Green, G. M., and Lyman, R. L. (1972): Feedback regulation of pancreatic enzyme secretion as a mechanism for trypsin inhibitor-induced hypersecretion in rats. Proc. Soc. Exp. Biol. Med., 140, 6-12.

21) Schneeman, B. O., Chang, I., Smith, L. B., and Lyman, R. L. (1977): Effect of dietary amino acids, casein, and soybean trypsin inhibitor on pancreatic protein secretion in rat. J. Nutr., 107, 281-288.

22) Green, G. M., Olds, B. A., Matthews, G., and Lyman, R. L. (1973): Protein, as a regulator of pancreatic enzyme secretion in the rat. Proc. Soc. Exp. Biol. Med., 142, 1162-1167.

23) Miyasaka, K., and Green, G. M. (1984): Effect of partial exclusion of pancreatic juice on rat basal pancreatic secretion. Gastroenterology, 8, 114-119.

24) Green, G. M., and Nasset, E. S. (1980): Importance of bile in regulation of intraluminal proteolytic enzyme activities in the rat. Gastroenterology, 79, 695-702.

25) Dooley, C. P., and Valenzuela, J. E. (1984): Duodenal volume and osmoreceptors in the stimulation of human pancreatic secretion. Gastroenterology, 86, 23-27. 